\title{
Optimization of the thin-walled rod with an open profile
}

\author{
Vladimir Andreev ${ }^{1,}{ }^{*}$ Elena Barmenkova ${ }^{1,2}$ \\ ${ }^{1}$ Moccow State University of Civil Engineering, Yaroslavskoye sh., Moscow 129110, Russia \\ ${ }^{2}$ Peoples' Friendship University of Russia, Miklukho-Maklaya str., Moscow 117198, Russia
}

\begin{abstract}
The purpose of this study is to develop optimization of the cross-section of the inhomogeneous thin-walled rod with an open profile compared to the traditionally used homogeneous. The idea of the developed method of optimization is to determine the law of variation of the elasticity modulus, at which the stress across the crosssection "aligned" and approaching to a constant value. Thus, it is possible to use all material of rod, which leads to optimization of its structure, i.e. an increase efficiency of rod.
\end{abstract}

\section{Introduction}

Due to the widespread of thin-walled structures in the construction practice, which characterized by reduced material consumption and weight, we substantiate the optimization method of such structures. A number of works on optimization of thinwalled open profile rod provides the variation of different parameters subject to the conditions of the problem of optimal design (for example, conditions of strength) [1-5]. In this paper we consider optimization method, which based on solving the inverse problem of the theory of elasticity of inhomogeneous bodies, the essence of which is to determine the law of changing the modulus of elasticity on the rod's height for which stress state will be given.

\section{Strength analyses}

Below are the results of the strength calculations of the roof run. The design scheme is presented in the form of simply-supported at its ends thin-walled rod (Fig. 1). As the profile of the rod is considered the steel channel, because it is the most profitable for the roof runs, the most desirable structurally and in terms of the convenience of attaching it to a belt truss (Fig. 2).

The initial data:

Length of the roof run: $l=600 \mathrm{~cm}$. Roof pitch: $\alpha=5^{\circ}$.

Design load on the run: $q=300 \mathrm{~kg} / \mathrm{m}$.

Geometric characteristics of the profile: №16P by GOST 8240-89:

\footnotetext{
* Corresponding author: asv@mgsu.ru
} 
$h=16 \mathrm{~cm}, b=6.4 \mathrm{~cm}, d=0.5 \mathrm{~cm}, t=0.84 \mathrm{~cm}, x_{0}=1.97 \mathrm{~cm}, W x=93.8 \mathrm{~cm}^{3}, W y \min =16.4$ $\mathrm{cm}^{3}, I_{x}=750 \mathrm{~cm}^{4}, I_{y}=72.8 \mathrm{~cm}^{4}$.

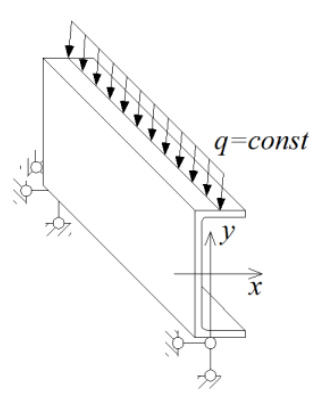

Fig.1. Design scheme.

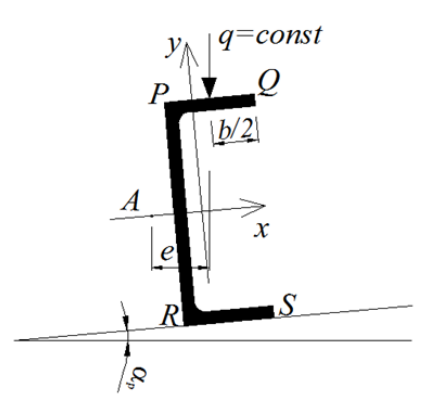

Fig.2. Channel cross section.

Characteristics of the material steel: $R_{\mathrm{y}}=240 \mathrm{MPa}, E=2.1 \cdot 10^{5} \mathrm{MPa}, G=$ $0.8 \cdot 10^{5} \mathrm{MPa}$.

The roof run, resting on an inclined plane and being under the influence of vertical loads, should be calculated on the combined effect of bending with torsion. So it is necessary to carry out the calculation of the strength of elements under the action of moments $\left(M_{x}, M_{y}\right)$ in the two principal planes and the presence of bimoment $\left(B_{\omega}\right)$ (paragraph 8.2.1 SP 16.13330.2011).

Bending moments in the two principal planes:

$$
M_{x}=\frac{q_{\mathrm{p}} \cdot l^{2}}{8} \cdot \sin \alpha_{\mathrm{p}}=117.66 \mathrm{kHcm} ; M_{y}=\frac{q_{\mathrm{p}} \cdot l^{2}}{8} \cdot \cos \alpha_{p}=1345 \mathrm{kHcm} .
$$

To determine the bimoment calculate the sectorial geometric section properties.

Sectorial coordinate:

$$
\omega_{0}=2 \cdot \frac{1}{2} \cdot\left(\frac{h}{2}-\frac{t}{2}\right) \cdot\left(b-\frac{d}{2}\right)=2 \cdot \frac{1}{2} \cdot 7.58 \cdot 6.15=46.62 \mathrm{~cm}^{2} .
$$

Coordinate of the torsion center:

$$
\alpha_{x}=-\frac{I_{\omega \mathrm{y}}}{I_{x}}=-2.43 \mathrm{~cm}
$$

Here $I_{\omega \mathrm{y}}=2 \cdot\left(\frac{1}{2} \cdot \omega_{0} \cdot\left(\frac{h}{2}-\frac{t}{2}\right) \cdot\left(b-\frac{d}{2}\right)\right) \cdot t=1825 \mathrm{~cm}^{5}-$ sector-linear moment of inertia (static moment).

The values of the main sectorial coordinate in the characteristic points of the cross section (Fig. 2):

lower the leftmost point of the profile

$\omega_{R}=-2 \cdot \frac{1}{2} \cdot\left(\frac{h}{2}-\frac{t}{2}\right) \cdot\left|\alpha_{x}\right|=-18.45 \mathrm{~cm}^{2}$.

lower rightmost point of the profile

$\omega_{S}=-2 \cdot \frac{1}{2} \cdot\left(\frac{h}{2}-\frac{t}{2}\right) \cdot\left|\alpha_{x}\right|+\left(\frac{h}{2}-\frac{t}{2}\right) \cdot\left(b-\frac{d}{2}\right)=28.17 \mathrm{~cm}^{2}$.

Sectorial moment of inertia: 


$$
I_{\omega}=2 \cdot\left[t \cdot \frac{\left(b-\frac{d}{2}\right)}{6} \cdot\left(2 \cdot \omega_{S}^{2}+2 \cdot \omega_{R}^{2}-2 \cdot \omega_{R} \cdot \omega_{S}\right)+d \cdot \frac{\left(\frac{h}{2}-\frac{t}{2}\right) \cdot \omega_{R}}{2} \frac{2}{3} \omega_{R}\right]=2975 \mathrm{~cm}^{6}
$$

Moment of inertia at free torsion considering factor for channel $\beta=1.12$ :

$$
I_{d}=\beta \frac{1}{3}\left(2 \cdot b \cdot t^{3}+h \cdot d^{3}\right)=3.58 \mathrm{~cm}^{4}
$$

Eccentricity (Fig. 2):

$$
e=\left(\left|\alpha_{\mathrm{x}}\right|+\frac{b}{2}-\frac{h}{2} \cdot \operatorname{tg} \alpha_{p}\right) \cdot \cos \alpha_{p}=4.92 \mathrm{~cm} .
$$

Bimoment taking into account the design scheme:

$$
B_{\omega}=\frac{q_{\mathrm{p}} \cdot e \cdot\left(\operatorname{ch}\left(\frac{k l}{2}\right)-1\right)}{k^{2} \cdot \operatorname{ch}\left(\frac{k l}{2}\right)}=320.71 \mathrm{kHcm}
$$

where $k=\sqrt{\frac{G \cdot I_{d}}{E \cdot I_{\omega}}}=0,021-$ flexural-torsional characteristics.

Figure 3 shows the following diagrams of normal stresses: $\sigma\left(M_{x}\right), \sigma\left(M_{y}\right)-$ diagrams of bending in the two principal planes, $\sigma_{\omega}$ - sectorial stresses diagram, $\sigma$ diagram of total stresses.
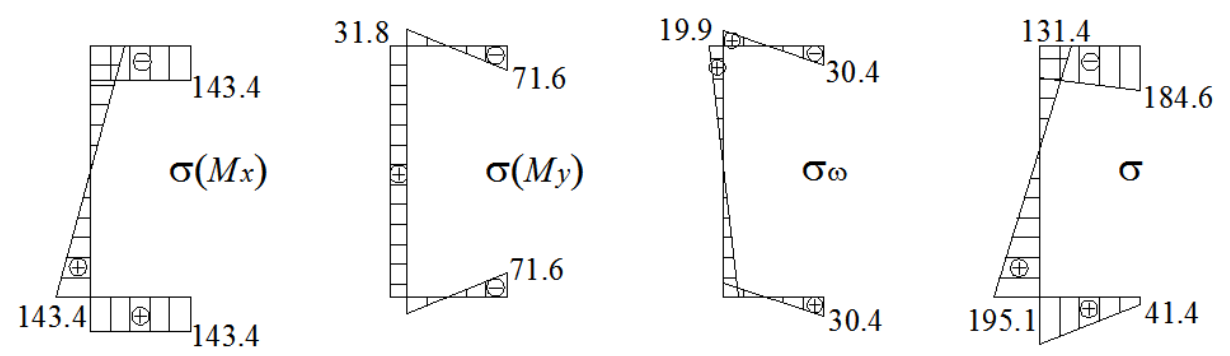

Fig. 3. The diagrams of normal stresses $\sigma\left(M_{x}\right), \sigma\left(M_{x}\right), \sigma_{\omega}, \sigma,[\mathrm{MPa}]$.

Thus, for the most strenuous point the value of total stress is: $\sigma_{\max }=195.1 \mathrm{MPa}$

The analysis of strength:

$$
\sigma_{\max }=195.1 \mathrm{MPa}<R_{y} \cdot \gamma_{c}=240 \cdot 0.9=216 \mathrm{MPa} \text {. }
$$

Strength is provided.

As can be seen from the results, for run channel profile account torsion gives reduction of normal stresses that is actually bimoment downloads profile, and it will be a reserve of strength. Thus, the strength analysis showed the strength of the rod is provided taking into account the unloading of profile of due to sectorial stress. 


\section{Stress state optimization method}

To optimize thin-walled open profile rods on torsion with bending will use the iterative method. As the initial solution, we take the solution for a homogeneous material, represented in the above paragraph 2. According to the presented stress diagram (Fig. 2) for a homogeneous rod, obviously, for equal-stress profile it is necessary to reduce the modulus of elasticity should be reduce as compared with a homogeneous material on the lower and upper fibers and increase at the neutral layer.

As a first approximation we consider the stress state of the rod, when the modulus of elasticity varies linearly:

$$
E^{(1)}(y)=E_{1}+\frac{2\left(E_{1}-E_{2}\right)}{h} y
$$

where $E_{1}=1.47 \cdot 10^{6} \mathrm{MPa}$ (the elastic modulus at $y=0$ ), $E_{2}=2.1 \cdot 10^{5} \mathrm{MPa}$ (the elastic modulus at $y=-h / 2$ ). Dependence of Young's modulus of the coordinate $y$ is searched in the range $(-h / 2,0)$, this is caused by the symmetry of the cross section and presented in point 2 strength analysis, according to which the lower-left point of the profile is the most strenuous.

Normal stresses $\sigma^{(1)}$ can be calculated by the formula:

$$
\sigma^{(1)}(y)=E^{(1)}(y)\left(\frac{\left(-M_{x}\right)}{\left[E I_{x}\right]_{1}} y+\frac{M_{y}}{\left[E I_{y}\right]_{1}} x-\frac{\left(-B_{\omega}\right)}{\left[E I_{\omega}\right]_{1}} \omega(y)\right) \text {. }
$$

Here

$$
\begin{aligned}
& {\left[E I_{x}\right]_{1}=2\left(\int_{-\left(\frac{h}{2}-t\right)}^{0} d \cdot E^{(1)}(y) \cdot y^{2} d y+\int_{-\frac{h}{2}}^{-\left(\frac{h}{2}-t\right)} b \cdot E^{(1)}(y) \cdot y^{2} d y\right)=2.5 \cdot 10^{7} \mathrm{kN} \cdot \mathrm{cm}^{2},} \\
& {\left[E I_{y}\right]_{1}=2\left(\int_{-\left(\frac{h}{2}-t\right)}^{0} E^{(1)}(y) \cdot\left[\int_{-x_{0}}^{-\left(x_{0}-d\right)} x^{2} d x\right] d y+\int_{-\frac{h}{2}}^{-\left(\frac{h}{2}-t\right)} E^{(1)}(y) \cdot\left[\int_{-x_{0}}^{b-x_{0}} x^{2} d x\right] d y\right)=3.4 \cdot 10^{6} \mathrm{kN} \cdot \mathrm{cm}^{2}-}
\end{aligned}
$$

the reduced bending stiffness,

$$
\left[E I_{\omega}\right]_{1}=2\left[\int_{-\left(\frac{h}{2}-\frac{t}{2}\right)}^{0} d \cdot E^{(1)}(y) \cdot\left(\frac{\omega_{P}}{\frac{h}{2}-\frac{t}{2}} y\right)^{2} d y+\frac{\left(\mathrm{b}-\frac{\mathrm{d}}{2}\right)}{6} \cdot\left(2 \cdot \omega_{S}^{2}+2 \cdot \omega_{R}^{2}-2 \cdot \omega_{R} \cdot \omega_{S}\right) \int_{-\frac{h}{2}}^{-\left(\frac{h}{2}-\frac{t}{2}\right)} E^{(1)}(y) d y\right]=
$$

$=1.1 \cdot 10^{8} \mathrm{kN} \cdot \mathrm{cm}^{4}-$ the sectorial stiffness,

On the next step of iteration process using condition $\sigma=$ const we define the function $E^{(2)}(y)$ :

$$
E^{(2)}(y)=\frac{\sigma}{\frac{\left(-M_{x}\right)}{\left[E I_{x}\right]_{1}} y+\frac{M_{y}}{\left[E I_{y}\right]_{1}} x-\frac{\left(-B_{\omega}\right)}{\left[E I_{\omega}\right]_{1}} \omega(y)} .
$$

Thus, we have obtained the second distribution of the elastic modulus on height of cross-section. Distribution $E^{(2)}(y)$ represented in the Table 1. 
Now we can define the stresses $\sigma=\sigma^{(2)}(y)$ by using formula for $E(y)=E^{(2)}(y)$

$$
\sigma^{(2)}(y)=E^{(2)}(y)\left(\frac{\left(-M_{x}\right)}{\left[E I_{x}\right]_{2}} y+\frac{M_{y}}{\left[E I_{y}\right]_{2}} x-\frac{\left(-B_{\omega}\right)}{\left[E I_{\omega}\right]_{2}} \omega(y)\right)
$$

Stiffness are increased in comparison with the values on the first step of iteration process:

$$
\begin{aligned}
& {\left[E I_{x}\right]_{2}=2\left(\int_{-\left(\frac{h}{2}-t\right)}^{0} d \cdot E^{(2)}(y) \cdot y^{2} d y+\int_{-\frac{h}{2}}^{-\left(\frac{h}{2}-t\right)} b \cdot E^{(2)}(y) \cdot y^{2} d y\right)=3.2 \cdot 10^{7} \mathrm{kN} \cdot \mathrm{cm}^{2},} \\
& {\left[E I_{y}\right]_{2}=2\left(\int_{-\left(\frac{h}{2}-t\right)}^{0} E^{(2)}(y) \cdot\left[\int_{-x_{0}}^{-\left(x_{0}-d\right)} x^{2} d x\right] d y+\int_{h}^{-\frac{h}{2}} E^{(2)}(y) \cdot\left[\int_{-x_{0}}^{b-x_{0}} x^{2} d x\right] d y\right)=4.22 \cdot 10^{6} \mathrm{kN} \cdot \mathrm{cm}^{2}} \\
& {\left[E I_{\omega}\right]_{2}=2\left(\int_{-\left(\frac{h}{2}-\frac{t}{2}\right)}^{0} d E^{(1)}(y) \cdot\left(\frac{\omega_{P}}{\frac{h}{2}-\frac{t}{2}} y\right)^{2} d y+\frac{\left(-\frac{h}{2}-\frac{t}{2}\right)}{6}\left(2 \omega_{S}{ }^{2}+2 \omega_{R}^{2}-2 \omega_{R} \omega_{S} \int_{-\frac{h}{2}}^{(1)}(y) d y\right)=\right.}
\end{aligned}
$$

$=1.3 \cdot 10^{8} \mathrm{kN} \cdot \mathrm{cm}^{4}$.

The function $E^{(3)}(y)$ is given by:

$$
E^{(3)}(y)=\frac{\sigma}{\frac{\left(-M_{x}\right)}{\left[E I_{x}\right]_{2}} y+\frac{M_{y}}{\left[E I_{y}\right]_{2}} x-\frac{\left(-B_{\omega}\right)}{\left[E I_{\omega}\right]_{2}} \omega(y)} .
$$

The expression for $\sigma^{(3)}(y)$ takes the following form:

$$
\sigma^{(3)}(y)=E^{(3)}(y)\left(\frac{\left(-M_{x}\right)}{\left[E I_{x}\right]_{3}} y+\frac{M_{y}}{\left[E I_{y}\right]_{3}} x-\frac{\left(-B_{\omega}\right)}{\left[E I_{\omega}\right]_{3}} \omega(y)\right) \text {. }
$$

\begin{tabular}{|c|c|c|c|c|c|c|c|c|}
\hline$y$, & $E^{(\mathbf{0})}$ & $\sigma^{(0)}$ & $E^{(1)}(y)$ & $\sigma^{(1)}$ & $E^{(2)}(y)$ & $\sigma^{(2)}$ & $E^{(3)}(y)$ & $\sigma^{(3)}$ \\
\hline m & \multicolumn{8}{|c|}{ MPa } \\
\hline 0 & \multirow{5}{*}{$2.4 \cdot 10^{5}$} & 31.2 & $1.47 \cdot 10^{6}$ & 100.3 & $3.16 \cdot 10^{6}$ & 173.8 & $3.93 \cdot 10^{6}$ & 169.5 \\
\hline-2 & & 72.8 & $1.16 \cdot 10^{6}$ & 222.0 & $1.12 \cdot 10^{6}$ & 169.9 & $1.43 \cdot 10^{6}$ & 168.9 \\
\hline-4 & & 114.3 & $8.4 \cdot 10^{5}$ & 265.5 & $6.83 \cdot 10^{5}$ & 169.1 & $8.73 \cdot 10^{5}$ & 168.8 \\
\hline-6 & & 155.9 & $1.16 \cdot 10^{6}$ & 231.1 & $4.91 \cdot 10^{5}$ & 168.7 & $6.28 \cdot 10^{5}$ & 168.9 \\
\hline-8 & & 195.1 & $2.1 \cdot 10^{5}$ & 118.5 & $3.83 \cdot 10^{5}$ & 168.5 & $4.91 \cdot 10^{5}$ & 168.8 \\
\hline
\end{tabular}

The calculation results are shown in Table 1. According results, it can be argued that in the considered problem will be enough execute three approximations.

Table 1. The values of the modulus of elasticity $E^{(i)}$ and the total stresses $\sigma^{(i)}$ for the stages of the iterative process. 
As seen from the results, taking into account inhomogeneity of the material leads to a substantial redistribution of the stresses in the structure. This redistribution has quantitative and qualitative character.

In the case of a homogeneous material stresses in the extreme fibers differ more than by 6.0 times, whence it follows that the profile is generally underloaded. In the inhomogeneous rod for the case $E(y)=E^{(3)}(y)$ stresses are constant over the cross section, that allows using all material of rod, which generally increases its efficiency.

To determine the effect of an optimized model of an inhomogeneous structure, we introduce the efficiency ratio of work of inhomogeneous beam: $\beta=\sigma_{\max }^{(\text {hom })} / \sigma_{\max }^{(\mathrm{inh})}[$ [11]. In formula $\sigma_{\max }^{(\text {hom })}$ и $\sigma_{\max }^{(\text {inh })}=$ const are respectively the maximum stresses in the homogeneous and inhomogeneous rods. In the above case, this factor is: $\beta=\sigma_{\text {max }}^{(0)} / \sigma_{\text {max }}^{(3)}=1.2$ (see Table 1).

The question naturally arises how to create a beam with a continuous change of its stiffness on height cross-section. We can offer a way to create a piecewise homogeneous structure, as was done in [7-11]. Thus, we come to the problem of multilayered structures. Mechanical characteristics of each layer of the multilayer structure are assigned from the solution of the inverse problem of determining the dependence of $E(y)$.

In this case, naturally, the coefficient $\beta$ for multilayer-rod will be smaller than for the beam with continuous inhomogeneity, but with an increasing number of layers it will be closer to the above value, which will give economic effect.

\section{Conclusions}

The obtained solution of problem for the channel profile at combined effect of bending with torsion gives justification to use the developed optimization method for thinwalled rod of various profiles.

The used method allows to improve the structure of thin-walled rod with the open profile under bending with torsion. The developed method of optimization leads to a significant reduction of stresses in inhomogeneous rod compared to the traditionally used homogeneous and the stresses over the cross section are approaching to a constant value. Thus, it is possible to use all material of rod, which leads to optimization of its structure, i.e. an increase efficiency of rod. The reduction in stresses in comparison with the homogeneous rod can increase the loads, reduce the size of the rod, so it will allow to receive some economic effect.

\section{Acknowledgment}

This work was financially supported by the Ministry of Russian Education (state task \#2014/14).

\section{References}

1. D.V. Bychkov, Structural Mechanics of thin-walled structures, (1962)

2. G.I. Grebenyuk, A.A. Gavrilov, E.V. Yankov, Proceedings of the universities. Building 7, 3-11 (2013)

3. T.L. Dmitrieva, Vestnik of Irkutsk State Technical University 5, 88-94 (2010) 
4. A.V. Izhendeev, International Research Journal 4(46), (2016)

5. Z. Freidman, M.D. Fuchs, Comput. and Structures 3(25), 405-414 (1987)

6. V.I. Andreev, Some problems and methods of mechanics of inhomogeneous bodies, (2002)

7. V.I. Andreev, I.A. Potekhin, Bulletin of the Department of Civil Engineering of the Russian Academy of Architecture and Building Sciences 11, 48-52 (2007)

8. V.I. Andreev, E.V. Barmenkova, A.V. Matveeva, Vestnik of Moscow State University of Civil Engineering 1, 25-32 (2014)

9. V.I. Andreev, E.V. Barmenkova, A.V. Matveeva, Applied Mechanics and Materials 501-504, 645-648 (2014)

10. V.I. Andreev, E.V. Barmenkova, Procedia Engineering 91, 20-25 (2014)

11. V.I. Andreev, E.V. Barmenkova, I. A. Potekhin, Procedia engineering 153, 37-44 (2016) 